\title{
Application of temperature modulation to FTIR spectroscopy: an analysis of equilibrium and non-equilibrium conformational transitions of poly(ethylene terephthalate) in glassy and liquid states
}

\author{
Valerio Di Lisio ${ }^{1}$ (D) Elisa Sturabotti ${ }^{1} \cdot$ Iolanda Francolini ${ }^{1} \cdot$ Antonella Piozzi $^{1} \cdot$ Andrea Martinelli $^{1}$
}

Received: 19 November 2019 / Accepted: 4 August 2020 / Published online: 28 August 2020

(c) The Author(s) 2020

\begin{abstract}
In this paper, the application of a temperature modulation to the temperature-resolved FTIR analysis is reported. The advantage offered by the spectroscopic investigation, able to follow the micro-structural and conformational sample modification involved in sample thermal transformation, was merged to that of temperature modulation, related to the possibility to separate the reversing (in-equilibrium within the experimental condition) to the non-reversing (non-equilibrium) processes. The potentiality of the technique (modulated temperature FTIR, MTFTIR) is highlighted through the study of the thermal transitions of amorphous poly(ethylene terephthalate) from $50{ }^{\circ} \mathrm{C}$ to the cold-crystallization. After the presentation of the theoretical framework and the experimental conditions, a step-by-step description of acquired data elaboration is given. The total variation of a selected band intensity as function of mean temperature as well as its reversing and non-reversing components are obtained. The evolution of the bands at 1340 and $971 \mathrm{~cm}^{-1}$, assigned to the trans conformation of the ethylenic unit and to the all-trans conformation of the repeating unit, respectively, are investigated. As expected, the glass transition is observed in the reversing components meanwhile the recovery of the glass relaxation and cold crystallization in non-reversing ones. Particularly interesting resulted the behaviour of the sample in the supercooled liquid state, between the glass transition and the cold-crystallization onset, in which the results show that the ethylenic conformers are in-equilibrium while the all-trans sequences are not. MTFTIR is confirmed to be a technique particularly suitable for the characterization of non-equilibrium conformational states of polymers.
\end{abstract}

Keywords MTFTIR · Modulated temperature $\cdot$ Poly (ethylene terephthalate) $\cdot$ Conformational rearrangement $\cdot$ Nonequilibrium transition $\cdot$ Liquid relaxation

\section{Introduction}

Thermal analysis is considered one of the main issues in materials characterization, both from the applicative and fundamental research point of view [1]. A comprehensive definition of material properties, investigated under different aspects, can be pursued and achieved successfully by combining different techniques, methods

Electronic supplementary material The online version of this article (https://doi.org/10.1007/s10973-020-10169-0) contains supplementary material, which is available to authorized users.

Valerio Di Lisio

valerio.dilisio@uniroma1.it

1 Department of Chemistry, Sapienza University of Rome, P.le A. Moro 5, 00185 Rome, Italy and experimental conditions [2]. In particular, the complex thermal transformations of polymers as well as the elaboration of complex interpretative models, to predict material properties, have been obtained [3]. In this contest, the differential scanning calorimetry is one of the most used analysis. An improvement of the technique was achieved in 1993 when the Modulated Temperature Differential Scanning Calorimetry (MTDSC) has been developed [4] by adding a periodic temperature perturbation of specified amplitude and frequency (commonly a sinusoidal function) to the conventional linear heating or cooling ramp. MTDSC offers the great advantage to identify and separate the reversing temperature-dependent heat response, that is reversed by temperature oscillation, from the non-reversing kinetic processes involved in material thermal transitions [4-19]. The study of several amorphous and semi-crystalline polymeric systems, including 
the most widespread poly(ethylene terephthalate) [8-10], poly(ethylene) [11, 12], isotactic poly(propylene) [10, 13, 14], poly(L-lactide) [15], has led to a deeper understanding of thermodynamics and kinetics phenomena involved in polymer vitrification $[9,16,17]$, crystallization $[10,18]$ and melting $[8,9,11-15]$ as well as of the role of rigid amorphous fraction on the thermal behaviour [19] of high molecular weight compounds.

Later on, a further implementation has been reached by the introduction of the Fast-Scan DSC (FSDSC) which allows the use of scan rates much higher than that of the conventional DSC or MTDSC [20]. This opened the possibility to investigate material thermal properties otherwise not accessible, including analyses of metastable states, timedependent transformation or transitions involving low heat flux [21]. Moreover, other types of temperature perturbations, such as that used in step response method, allowed to measure the periodic heat response of the system not only at the base frequency of oscillation, but also at the higher harmonics $[22,23]$. In this way, the FSDSC has extended the temperature modulation frequency from $10^{-3}$ to $10^{-1} \mathrm{~Hz}$ of conventional MTDSC, to $10^{-1}-10^{2} \mathrm{~Hz}$. The interpretative advantages of the experiments based on temperature modulation have been successfully exploited also for other investigation techniques, such as thermomechanical analysis [24], ellipsometry [25], dynamic mechanical analysis [26], thermogravimetry [27], small- and wide-angle X-ray scattering [28, 29], optical microscopy [30] and refractometry [31]. However, unlike calorimetric, mechanical and optical techniques, which follow only one signal output, namely heat flow, storage and loss modulus, sample dimension or mass, refractive index (mass density) or transmitted light intensity, the thermal analysis carried out by spectroscopic and diffractometric techniques such as temperature-time resolved $\mathrm{X}$-ray diffraction and FTIR allows to obtain a multi-signal investigation. The infrared spectroscopy, in fact, being sensitive to the changes of inter- or intra-molecular interactions, conformation or structure, allows to characterize materials at a molecular level during sample thermal transformations. Therefore, by following intensity variations of different absorption bands as a function of time or temperature, transition details, such as secondary crystallizations involving low heat fluxes [32], mesophase formation and melting [33], conformer concentration variation [34], polymorphic transitions [35] and other features not easily or not detectable by conventional thermal analyses, can be studied. Modulated stimuli were applied also to infrared spectroscopy by changing periodically temperature, $\mathrm{pH}$, or concentration around a constant value [36]. For example, the temperaturemodulated excitation infrared (T-MEIR) spectroscopy was used to investigate conformational and structural changes of poly(L-lysine) by an accurate separation of overlapped absorption bands [37].
In recent years, the study of non-equilibrium properties of polymers has become a fundamental issue in relation to those processing protocols that do not allow the liquid state of polymers to reach the equilibrium. In this framework, investigations on non-equilibrium molecular conformations is an important issue to relate macromolecular microscopic aspects to macroscopic properties of polymer products (e.g. mechanical strength, viscosity, thermal expansion) [38]. As a result of this demand, an implementation of variable temperature FTIR spectroscopy, obtained by the superimposing to the conventional linear heating ramp a temperature modulation (modulated temperature FTIR, MTFTIR $[39,40])$, was designed. While the sample was subject to the temperature program, a series of spectra were acquired sequentially and the intensities of selected absorption bands measured as function of time and temperature. By processing the oscillating temperature and intensity by an algorithm based on Sliding Fast Fourier Transform (SFFT), the total (mean) intensity variations as well as its reversing and nonreversing components as a function of the mean temperature were obtained for each analysed band. The reversing intensity is related to the sample transformation, such as conformational equilibria, that can be reversed within the employed temperature modulation amplitude and period, while the non-reversing component, to the spontaneous processes that cannot be reversed during one cycle of modulation (e.g. conformational or structural relaxations, phase transitions).

Poly(ethylene terephthalate) (PET) is the material chosen for this study because it has been largely investigated as a standard polymer for modulated temperature protocols [8-10, 19] thanks to the possibility to obtain samples in amorphous state easily and, so, to follow the main transitions in the range of $25-300{ }^{\circ} \mathrm{C}$, suitable for most thermal analysis techniques. Moreover, the comprehensive study of macro- and microscopical properties of PET involved in thermal transitions have been extensively studied by different thermal analyses [34, 41-47]. Infrared spectroscopy is a suitable technique able to study the complex conformational and structural behaviour of PET [34, $41,42]$, characterized by a backbone that allow many rotational degrees of freedom. In fact, the mid-infrared spectrum of PET is characterized by several absorption bands related to the possible conformations the macromolecules can adopt through the internal rotation about three bonds of the repeating unit [48]. The $\mathrm{CH}_{2}-\mathrm{CH}_{2}$ ethylenic bond of the ethylene glycol moiety and the $\mathrm{O}-\mathrm{CH}_{2}$ glycolic bond can be found in trans and gauche conformations ( $\mathrm{T}$ or $\mathrm{G}$ and $\mathrm{t}$ or $\mathrm{g}$, respectively), while the terephthalic unit can adopt two energetically equivalent isomeric state, cis $\left(\mathrm{C}_{\mathrm{B}}\right)$ and trans $\left(\mathrm{T}_{\mathrm{B}}\right)$, by rotation about the $\mathrm{O}=\mathrm{C}-\mathrm{C}_{\text {aryl }}$ bond [48-51]. Actually, other studies inferred that the unit can be found also in non-planar arrangement due to the little energetic barrier between the two forms $[52,53]$. In the crystalline state the PET chains assumed an all-trans structure 
$\left(\mathrm{TtT}_{\mathrm{B}}\right)$ [54], meanwhile in the amorphous state the gauche ethylenic $(\mathrm{G})$ and glycolic trans $(\mathrm{t})$ conformers are energetically favourite $[48,49]$.

The MTFTIR experiment was performed on quenched amorphous poly(ethylene terephthalate), heated by the oscillating temperature program in the glass transition and coldcrystallization temperature range. The conformational changes involved in sample thermal transformation has been detected by following the intensity variations of two selected absorption bands. In particular, the study was focused on the analysis of the bands centred at $1340 \mathrm{~cm}^{-1}$, assigned to the trans isomer of the glycolic unit (T) [34, 49-51], and at $971 \mathrm{~cm}^{-1}$, due to the all-trans sequence $\left(\mathrm{TtT}_{\mathrm{B}}\right)$ of the PET repeating unit $[42$, 49-51].

The paper is structured in four parts. In the first, a brief theoretical background about the effect of the temperature modulation on the infrared spectroscopic signals was given. Then, in the Experimental section, the employed temperature program, the spectra acquisition conditions and the modulated data elaboration were reported. In a subsection of the Results, the intensity variation of the band at $1340 \mathrm{~cm}^{-1}$ was used to show step-by-step the results of the data elaboration. Finally, the comparison of the total intensity variation as well as its reversing and non-reversing components of the two selected bands as a function of the mean temperature was discussed. The different MTFTIR behaviour of the the two bands in the supercooled liquid region, between the $T_{\mathrm{g}}$ and the cold-crystallization onset, evidenced new features on the conformational behavior involved in polymer transformations, otherwise not accessible by other conventional thermal analyses.

\section{Theoretical background}

In a MTFTIR experiment the sample was subjected to a modulated temperature protocol, $T(t)$, comprised of a linear heating ramp, that defines the mean temperature of the experiment $T^{\mathrm{M}}(t)$, with initial temperature $T_{0}$ and heating rate $b$, and a superimposed temperature oscillation, $\tilde{T}(t)$. By employing a sinusoidal temperature modulation with an amplitude $A_{\mathrm{T}}$ and an angular frequency $\omega$, the actual sample temperature $(T)$, the mean temperature $\left(T^{\mathrm{M}}\right)$ and the instantaneous heating rate $(\mathrm{d} T / \mathrm{d} t)$ as a function of time could be written as

$T(t)=T^{\mathrm{M}}+\tilde{T}=T_{0}+b t+A_{\mathrm{T}} \sin \omega t$

$T^{\mathrm{M}}(t)=T_{0}+b t$

$\frac{\mathrm{d} T}{\mathrm{~d} t}=b+A_{\mathrm{T}} \omega \cos \omega t$

Analogously to the general equation which describe the calorimetric response of a system under temperature perturbation $[4,6,7]$, the intensity change with time of a generic absorption band can be considered as the sum of a thermodynamic term, dependent only on the temperature of the system, and a kinetic term:

$\frac{\mathrm{d} I_{\overline{\mathrm{v}}}}{\mathrm{d} t}(t, T, x)=\frac{\mathrm{d} I_{\overline{\mathrm{v}}}^{\mathrm{R}}}{\mathrm{d} T} \frac{\mathrm{d} T}{\mathrm{~d} t}(T)+f(t, T, x)$

where $I_{\bar{\nu}}$ is the overall intensity calculated at the wavenumber $\bar{\nu}, I_{\bar{\nu}}^{\mathrm{R}}$ is the reversing intensity, that is the part of the overall intensity that depends only on the temperature of the system and $f(t, T, x)$ is a generic kinetic function dependent on time, temperature and extent of transition $x$. By introducing the modulated temperature protocol (Eq. 1) in Eq. 4 and by considering the linear response of the system $[6,7]$, the equation that describe the overall intensity variation with time is

$\frac{\mathrm{d} I_{\overline{\mathrm{v}}}}{\mathrm{d} t}=\frac{\mathrm{d} I_{\bar{\nu}}^{\mathrm{R}}}{\mathrm{d} T} b+f^{\mathrm{M}}\left(t, T^{\mathrm{M}}, x^{\mathrm{M}}\right)+\frac{\mathrm{d} I_{\bar{\nu}}^{\mathrm{R}}}{\mathrm{d} T} A_{\mathrm{T}} \omega \cos \omega t+\frac{\partial f^{\mathrm{M}}}{\partial T} A_{\mathrm{T}} \sin \omega t$

where $f^{\mathrm{M}}\left(t, T^{\mathrm{M}}, x^{\mathrm{M}}\right)$ is the average kinetic function depending on time, mean temperature and mean extent of transition. The first two terms of Eq. 5 are independent from the temperature modulation, meanwhile the third and fourth terms are in-phase and out-of-phase respect to the instantaneous heating rate, respectively (Eq. 3). It is worth to evidence the strict similarity of signal responses between the calorimetric heat flow and spectroscopic band intensity acquired in modulated temperature experiments, in which $\frac{\mathrm{d} I_{i}^{R}}{\mathrm{~d} T}$ can be considered analogous to the temperature dependent heat capacity of the system $\left(C=\frac{\mathrm{d} Q}{\mathrm{~d} T}\right)$ [7].

The overall intensity response $I_{\bar{\nu}}$ as function of time is given by the sum of the band intensity at the start of the experiment, $I_{\bar{\nu} 0}$, and the intensity variation $\Delta I_{\bar{\nu}}$ recorded during the modulated temperature program. It is obtained by integrating the Eq. 5 with respect to time:

$$
\begin{aligned}
I_{\bar{\nu}}(t)= & I_{\bar{\nu} 0}+\Delta I_{\bar{\nu}}(t)=I_{\bar{\nu} 0}+\int_{0}^{t} \frac{\mathrm{d} I_{\overline{\mathcal{L}}}^{\mathrm{R}}}{\mathrm{d} T} b \mathrm{~d} t+\frac{\mathrm{d} I_{\bar{\nu}}^{\mathrm{R}}}{\mathrm{d} T} A_{\mathrm{T}} \sin \omega t \\
& +\int_{0}^{t} f^{\mathrm{M}}\left(t, T^{\mathrm{M}}, x^{\mathrm{M}}\right) \mathrm{d} t-\frac{A_{\mathrm{T}}}{\omega} \frac{\partial f^{\mathrm{M}}}{\partial T} \cos \omega t
\end{aligned}
$$

The high-order terms, that could appear from the integration of the last term of Eq. 5 and that depends on the kinetic function $f^{\mathrm{M}}\left(t, T^{\mathrm{M}}, x^{\mathrm{M}}\right)$, were neglected.

The mean intensity, hereinafter called total intensity $\left(I_{\bar{\nu}}^{\mathrm{Tot}}\left(T^{\mathrm{M}}\right)\right)$, is calculated by averaging $I_{\bar{\nu}}(t)$ over one period of modulation. By using a change-of-variable from time to mean temperature, according to Eq. 2, it reads as 


$$
\begin{aligned}
I_{\bar{\nu}}^{\mathrm{Tot}}\left(T^{\mathrm{M}}\right) & =I_{\bar{\nu} 0}+\int_{T_{0}}^{T^{\mathrm{M}}} \frac{\mathrm{d} I_{\overline{\mathrm{\nu}}}^{\mathrm{R}}}{\mathrm{d} T} \mathrm{~d} T^{M}+\frac{1}{b} \int_{T_{0}}^{T^{\mathrm{M}}} f^{\mathrm{M}}\left(t, T^{\mathrm{M}}, x^{\mathrm{M}}\right) \mathrm{d} T^{\mathrm{M}} \\
& =I_{\bar{\nu} 0}+I_{\bar{\nu}}^{\mathrm{R}}+I_{\bar{\nu}}^{\mathrm{NR}}
\end{aligned}
$$

It's important to note that the total intensity is the same as would be obtained by a Variable Temperature FTIR experiment without modulation, with the same linear heating rate $b$ of the modulated program. The second term of the middle expression of Eq. 7 corresponds to the thermodynamic contribution to the total intensity variation, that is the reversing intensity $I_{\bar{\nu}}^{R}$. The third term, instead, represents the kinetic contribution and is defined non-reversing intensity $I_{\bar{\nu}}^{\mathrm{NR}}$.

In order to separate the thermodynamic and kinetic contributions from the total intensity described by Eq. 6, the deconvolution procedure proposed by Reading [7] and based on Fourier Transform was adapted to the MTFTIR analysis.

The third and fifth terms of Eq. 6 represent the modulated intensity response $\widetilde{\Delta I}_{\bar{\nu}}(t)$ to the temperature modulation $\tilde{T}(t)=A_{\mathrm{T}} \sin \omega t:$

$\widetilde{\Delta I}_{\bar{\nu}}(t)=\frac{\mathrm{d} I_{\bar{\nu}}^{\mathrm{R}}}{\mathrm{d} T} A_{\mathrm{T}} \sin \omega t-\frac{A_{\mathrm{T}}}{\omega} \frac{\partial f^{\mathrm{M}}}{\partial T} \cos \omega t$

The first sine term contains the thermodynamic response of the system in-phase respect to the temperature modulation, meanwhile the second cosine term is the out-of-phase kinetic response. The ratio between the modulated intensity response and modulated temperature $\left(\widetilde{\Delta I}_{\bar{\nu}} / \tilde{T}\right)$ could be expressed as the ratio between the frequency dependent complex intensity $I_{\bar{\nu}}^{*}$ and the complex temperature $T^{*}$ calculated at the angular frequency of the oscillation $\omega$. By applying the Fourier Transform, the complex ratio could be additionally expressed in terms of intensity and temperature modulation amplitudes ( $A_{\mathrm{I}_{\bar{\nu}}}$ and $A_{\mathrm{T}}$ respectively) as well as of the phase lag $\varphi$ between them:

$\frac{I_{\overline{\mathrm{V}}}^{*}}{T^{*}}(\omega)=\frac{A_{\mathrm{I}_{\overline{\mathrm{v}}}}}{A_{\mathrm{T}}} \cos \varphi-i \frac{A_{\mathrm{I}_{\overline{\mathrm{v}}}}}{A_{\mathrm{T}}} \sin \varphi=\frac{\mathrm{d} I_{\overline{\mathrm{V}}}^{\mathrm{R}}}{\mathrm{d} T}(\omega)+i \frac{1}{\omega} \frac{\partial f^{\mathrm{M}}}{\partial T}(\omega)$

By replacing the real and imaginary part of the Fourier Transform with the in-phase and out-of-phase coefficients found in Eq. 8, the last expression of Eq. 9 is obtained. Hence, by integrating the real part of Eq. 9 with respect to the mean temperature, the reversing intensity can be expressed as:

$I_{\bar{\nu}}^{\mathrm{R}}\left(\omega, T^{\mathrm{M}}\right)=\int_{T_{0}}^{T^{\mathrm{M}}} \frac{A_{I_{\bar{v}}}}{A_{\mathrm{T}}} \cos \varphi \mathrm{d} T^{\mathrm{M}}$

Ultimately, the non-reversing intensity $I_{\bar{\nu}}^{\mathrm{NR}}$ could be calculated by subtracting the two terms $I_{\bar{\nu}}^{\mathrm{R}}$ and $I_{\bar{\nu} 0}$ from $I_{\bar{\nu}}^{\mathrm{Tot}}$, according to Eq. 7. However, it is worth to mention that the elaboration procedure proposed herein leads to some interpretative problems of the non-reversing intensity generated during some transformations i.e. the glass transition, as pointed out by Schawe [6]. In fact, the non-reversing intensity is calculated by the difference between two quantities, $I_{\bar{\nu}}^{\text {Tot }}$ and $I_{\bar{\nu}}^{\mathrm{R}}$, that depend on two different time scales, the linear heating rate and the modulation frequency, respectively. For this reason, $I_{\bar{\nu}}^{\mathrm{NR}}$ can be approximated to the kinetic response of the system only when relaxation phenomena are slower than the time scale of the perturbation (i.e. for nonisothermal sub- $T_{\mathrm{g}}$ aging processes [39]).

\section{Experimental}

\section{Sample preparation}

Poly(ethylene terephthalate) $\left(M_{\mathrm{v}}=18 \mathrm{~kg} \mathrm{~mol}^{-1}\right)$, potassium bromide for IR spectroscopy, trifluoroacetic acid (TFA, purity $>95 \%$ ) and chloroform were purchased from Sigma Aldrich. FTIR transmission spectra were recorded on PET films, spin-coated on $\mathrm{KBr}$ pressed disks. A $2.5 \% \mathrm{w} / \mathrm{v}$ solution was prepared by dissolving the polymer in a $\mathrm{CHCl}_{3} /$ TFA 10:1 v/v mixture and casted $(50 \mu \mathrm{L})$ on the $\mathrm{KBr}$ disk by a PWM32 spin-coater (Headway Research) at $2000 \mathrm{rpm}$ for $120 \mathrm{~s}$. After vacuum drying, the films had a thickness of $5 \pm 1 \mu \mathrm{m}$. In order to achieve the amorphous polymer, the films were melted at $280{ }^{\circ} \mathrm{C}$ for $3 \mathrm{~min}$ and quenched in liquid nitrogen. Actually, it has been observed that cooling rate higher than about $3 \mathrm{~K} \mathrm{~s}^{-1}$ are necessary to avoid sample crystallization [55]. Then, to check the quenching procedure, a sample quenched in liquid nitrogen was also characterized by DSC (the DSC curve is reported in Online Resource 1). By comparing the experimental specific heat capacity variation at $T_{\mathrm{g}}\left(\Delta C_{\mathrm{p}}=0.390 \mathrm{~J} \mathrm{~g}^{-1} \mathrm{~K}^{-1}\right)$ with that expected from a completely amorphous sample $\left(\Delta C_{\mathrm{p}}^{0}=0.405 \mathrm{~J} \mathrm{~g}^{-1} \mathrm{~K}^{-1}[56]\right)$ and the enthalpy of cold crystallization $\left(\Delta H_{\mathrm{cc}}=-41.7 \mathrm{~J} \mathrm{~g}^{-1}\right)$ with that of the melting process $\left(\Delta H_{\mathrm{m}}=47.6 \mathrm{~J} \mathrm{~g}^{-1}\right)$, a residual crystallinity of about $5 \%$ could be inferred, a result analogous to that reported in literature for PET samples quenched in liquid nitrogen [45] and in water-ice bath [50, 51]. To eliminate any stresses caused by rapid quenching, all samples were heated at $82{ }^{\circ} \mathrm{C}$ for $5 \mathrm{~min}$, slight above the glass transition temperature $\left(T_{\mathrm{g}} \cong 80{ }^{\circ} \mathrm{C}\right)$. Then, the quenched samples were cooled to $50^{\circ} \mathrm{C}$ at $2{ }^{\circ} \mathrm{C} \mathrm{min}{ }^{-1}$ and immediately analysed with the modulated temperature protocol. Three repetitions of the MTFTIR experiment were made using different quenched PET samples in order to verify the reproducibility of the results. Although it has been reported that different glass structures could exist, depending on the specific cooling rate experienced by each sample [55], it was 
observed that all the quenched samples showed the same FTIR spectra.

\section{Temperature protocol and measurement}

The sample was placed in a SPECAC heating cell suitable for transmission FTIR analysis, equipped with a HELLMA thermo-programmer. The imposed temperature protocol consisted in a 4-segments program comprised of heating/isotherm/cooling/isotherm steps, repeated from 50 to $140{ }^{\circ} \mathrm{C}$. In each period, in the first segment the temperature was increased by $10{ }^{\circ} \mathrm{C}$ in $3 \mathrm{~min}$ (heating at $3.33^{\circ} \mathrm{C} \mathrm{min}^{-1}$ ), followed by a $1 \mathrm{~min}$ isotherm. Then, the temperature was decreased by $8{ }^{\circ} \mathrm{C}$ in $3 \mathrm{~min}$ (cooling at $2.66^{\circ} \mathrm{C} \mathrm{min}^{-1}$ ) and kept constant for $1 \mathrm{~min}$. However, the thermal inertia and conductivity as well as and heat dispersions of the heating cell brought about a large temperature lag between the sample and the programmed set-point. Hence, the sample temperature was measured by a K-thermocouple next to the infrared beam in contact with the PET film. Indeed, the actual heating rate resulted to be $0.20{ }^{\circ} \mathrm{C} \mathrm{min}^{-1}$, slight lower to that programmed. Moreover, the thermal inertia led to the smoothing of the sample temperature profile, that resulted to be well described by a sinusoidal function with an amplitude of $2.5 \pm 0.2{ }^{\circ} \mathrm{C}$ and a $480 \pm 5 \mathrm{~s}$ period. The comparison between the sample temperature, the programmed temperature and its sinusoidal fitting were reported in Online Resource 1 . To validate the accuracy of the sample temperature and the phase-lag of the modulated response, the melting of biphenyl $\left(T_{\mathrm{m}}=71^{\circ} \mathrm{C}\right)$ and benzoic acid $\left(T_{\mathrm{m}}=122^{\circ} \mathrm{C}\right)$ dispersed in $\mathrm{KBr}$ were followed by FTIR, using the modulated temperature protocol described above and already used in a previous study [39]. A sample temperature accuracy of $\pm 0.5{ }^{\circ} \mathrm{C}$ and a zero phase-lag between the temperature and spectral response in the absence of phase transitions were observed.

\section{Infrared spectra acquisition}

Infrared spectra were acquired sequentially during the modulated temperature program, using a Nicolet 6700 FTIR Spectrometer. Spectra were collected as function of time and temperature between 4000 and $400 \mathrm{~cm}^{-1}$ with a resolution of $4 \mathrm{~cm}^{-1}$ and by co-adding 40 scans per spectrum, corresponding to a sampling rate of one spectrum each $30 \mathrm{~s}$. Hence, 16 spectra were recorded for each period of modulation $(p=480 \mathrm{~s})$. At the end of the experiment, the acquisition time and temperature were associated to each spectrum and the integrated intensities of the bands centered at $1340 \mathrm{~cm}^{-1}\left(I_{1340}\right)$ and at $971 \mathrm{~cm}^{-1}\left(I_{971}\right)$ were measured, given a two point linear baseline intersecting the spectrum at $1354-1329 \mathrm{~cm}^{-1}$ and $993-947 \mathrm{~cm}^{-1}$, respectively.

\section{MTFTIR data elaboration}

The data elaboration comprised of three different steps aimed to obtain the total intensity variation $\left(I_{\bar{\nu}}^{\text {Tot }}\right)$ as a function of the mean temperature and its reversing $\left(I_{\bar{\nu}}^{\mathrm{R}}\right)$ and nonreversing $\left(I_{\bar{\nu}}^{\mathrm{NR}}\right)$ components. Firstly, the mean temperature $T^{\mathrm{M}}$ (Eq. 2) and the total intensity $I_{\bar{\nu}}^{\text {Tot }}$ (Eq. 7) were calculated by averaging $T(t)$ and $I_{\bar{\nu}}(t)$ over a limited data window of 16 values, corresponding exactly to one period of modulation. The so-called sliding average is repeated into the whole data range by shifting the averaging data window in order to eliminate the oscillating component. Subsequently, the oscillating functions of temperature $\tilde{T}(t)$ and intensity $\widetilde{\Delta I}_{\bar{\nu}}(t)$ were calculated by subtracting the averaged values $I_{\bar{\nu}}^{\text {Tot }}$ and $T^{\mathrm{M}}$ from $I_{\bar{\nu}}$ and $T$, respectively.

The second step of the elaboration procedure provided the calculation of the temperature and intensity modulation amplitudes $\left(A_{\mathrm{I}_{\overline{\mathrm{v}}}}, A_{\mathrm{T}}\right)$ as well as the phase lag $(\varphi)$ between them by means of the Sliding Fast Fourier Transform (SFFT). In this step it is mandatory to employ input signals that oscillates about a constant value, otherwise mathematical artifacts in the output signals was observed. The SFFT consists in a sequence of FFT calculated by the same process used for the sliding average. The calculation was performed on the modulated signals, $\widetilde{\Delta I}_{\bar{\nu}}(t)$ and $\tilde{T}(t)$, over a limited window of $n$ data sampled in one period of modulation $(n=16$ in the employed experimental conditions).

$$
\begin{aligned}
& I_{\bar{\nu}}^{*}(\omega)=\sum_{\mathrm{k}=1}^{n} \widetilde{\Delta I}_{\bar{\nu}}\left(t_{\mathrm{k}}\right) \cos \omega t_{\mathrm{k}}-i \sum_{\mathrm{k}=1}^{n} \widetilde{\Delta I}_{\bar{\nu}}\left(t_{\mathrm{k}}\right) \sin \omega t_{\mathrm{k}} \\
& T^{*}(\omega)=\sum_{\mathrm{k}=1}^{n} \tilde{T}\left(t_{\mathrm{k}}\right) \cos \omega t_{\mathrm{k}}-i \sum_{\mathrm{k}=1}^{n} \tilde{T}\left(t_{\mathrm{k}}\right) \sin \omega t_{\mathrm{k}}
\end{aligned}
$$

where $t_{\mathrm{k}}$ is the sampling time which must be equally spaced and follows the law $t_{\mathrm{n}=16}-t_{1}=p$.

By moving the window into the whole data range, the complex intensity $I_{\bar{\nu}}^{*}(\omega)$ and temperature $T^{*}(\omega)$ as a function of time or mean temperature, were obtained. In the present elaboration, only complex values calculated at the base frequency $\omega=\frac{2 \pi}{p}$ were considered, being the contribute of higher harmonics neglectable. Then, the temperature and intensity amplitudes as well as the phase lag were obtained as the moduli and arguments of the real and imaginary components of Eqs. 11 and 12, calculated according Eqs. 13, 14 and 15:

$$
\begin{aligned}
& A_{\mathrm{I}_{\bar{\nu}}}=\sqrt{\operatorname{Re}^{2}\left(I_{\bar{\nu}}^{*}\right)+\operatorname{Im}^{2}\left(I_{\bar{\nu}}^{*}\right)} \\
& A_{\mathrm{T}}=\sqrt{\operatorname{Re}^{2}\left(T^{*}\right)+\operatorname{Im}^{2}\left(T^{*}\right)}
\end{aligned}
$$


$\varphi=\tan ^{-1}\left(\frac{\operatorname{Im}\left(I_{\overline{\mathrm{V}}}^{*}\right)}{\operatorname{Re}\left(I_{\overline{\mathrm{v}}}^{*}\right)}\right)-\tan ^{-1}\left(\frac{\operatorname{Im}\left(T^{*}\right)}{\operatorname{Re}\left(T^{*}\right)}\right)$

The last step of the elaboration provided the calculation of the reversing intensity $I_{\bar{\nu}}^{\mathrm{R}}\left(T^{\mathrm{M}}\right)$ by means of the integration with respect to the mean temperature (Eq. 10) of the ratio $A_{\mathrm{I}_{\mathrm{v}}} / A_{\mathrm{T}}$, multiplied for the cosine of $\varphi$. Finally, the nonreversing intensity $I_{\bar{\nu}}^{\mathrm{NR}}\left(T^{\mathrm{M}}\right)$ was obtained by subtracting $I_{\bar{\nu}}^{\mathrm{R}}$ and $I_{\bar{\nu} 0}$ from $I_{\bar{\nu}}^{\text {Tot }}$.

\section{Results and discussion}

Sample temperature $T(t)$, mean temperature $T^{\mathrm{M}}$ and modulated temperature $\tilde{T}$ are reported as a function of the acquisition time in Fig. 1. The selected 4-segment temperature program, imposed by the controller, brought about an actual sinusoidal temperature modulation recorded by the K-thermocouple in contact with the sample, as shown in Online Resource 1.

During the modulated heating program, the spectra were acquired every $30 \mathrm{~s}$. In Fig. 2, an exemplificative collection of spectra, comprising of 1 spectrum per period, was reported as function of the sample temperature in two spectral windows, the $1430-1320 \mathrm{~cm}^{-1}$ (Fig. 2a) and $1070-940 \mathrm{~cm}^{-1}$ ranges (Fig. 2b).

In order to show the sequential steps used for the data elaboration, the integrated intensity of the selected band at $1340 \mathrm{~cm}^{-1}$ as function of time (Fig. 2a) was chosen as an example. This vibration is assigned to the $\mathrm{CH}_{2}$ wagging mode of PET ethylenic group in trans conformation $(T)$ [34, 49-51]. The overall intensity $I_{1340}$ as well as the mean $I_{1340}^{\mathrm{Tot}}$ and modulated intensity $\widetilde{\Delta I}_{1340}$ are reported as a function of



Fig. 1 Measured sample temperature (red solid line) and its separation into mean (blue dash-dot line) and modulated temperature (black solid line, bottom panel) as function of time
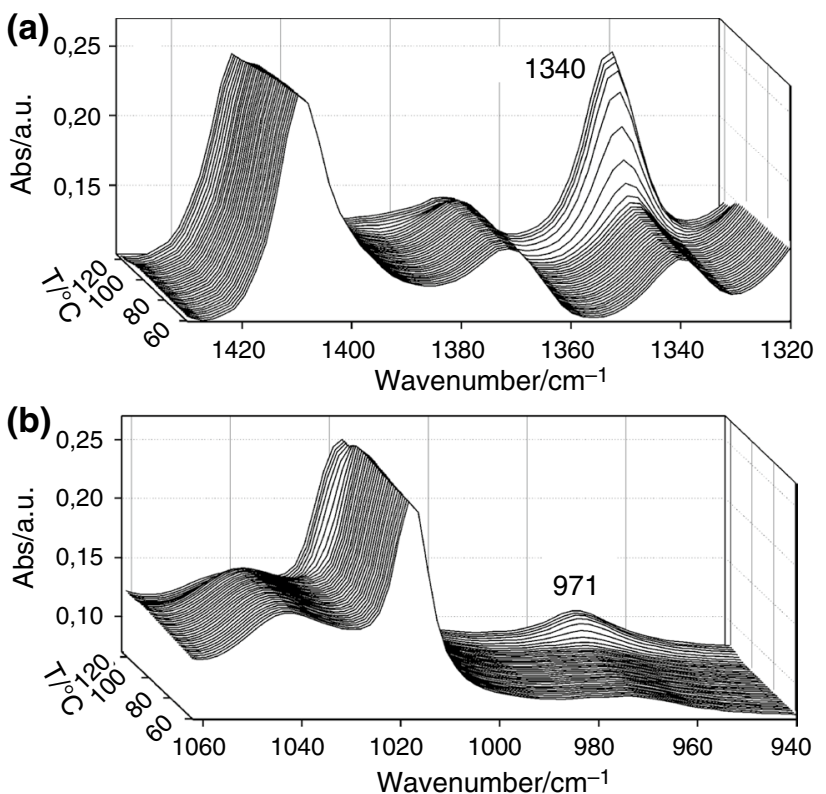

Fig. 2 FTIR transmission spectra of quenched PET acquired during the modulated temperature program as function of the sample temperature. One spectrum for each modulation is visualized in spectral ranges of $1430-1320 \mathrm{~cm}^{-1}$ (a) and $1070-940 \mathrm{~cm}^{-1}$ (b)

mean temperature in Fig. 3. The employed raw dataset of temperatures and band intensities, as well as modulated and mean signals can be found in Online Resource 2.

At a first glance, two main features can be observed. $I_{1340}$ and $I_{1340}^{\text {Tot }}$ show a steep increase between 100 and $110{ }^{\circ} \mathrm{C}$ due to the polymer cold-crystallization. It occurs, in fact, through the growth of all trans conformer sequences to form a ribbon-like chain structures which arranges in the crystal

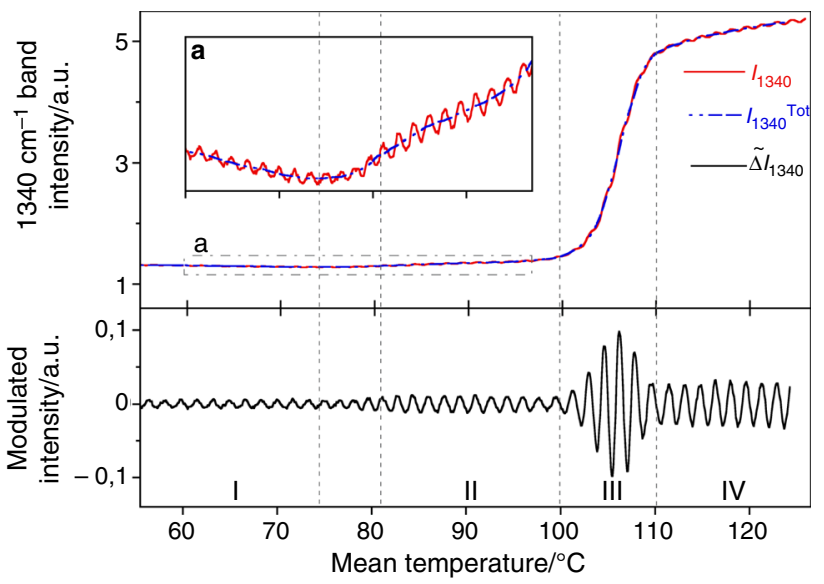

Fig. 3 Overall intensity of the band at $1340 \mathrm{~cm}^{-1}$ (red solid line, upper panel) and its total (blue dash-dot line, upper panel) and modulated (black solid line, bottom panel) components of the quenched PET sample as function of mean temperature. In the inset a the $y$-axis enlargement is reported to better highlight the glass transition 
lattice [54]. Moreover, at about $80{ }^{\circ} \mathrm{C}$, the negative to positive slope change of the total intensity variation with time highlights the glass transition. In Fig. 3a, the $y$-axis enlargement is reported to better evidence the transition.

The modulated intensity component contains the thermodynamics and kinetics information of the sample spectral transformations. For the sake of simplicity, the explored temperature range in Fig. 3 was divided in four regions according to the change of $\widetilde{\Delta I}_{1340}$ oscillation amplitude. Between 50 and $75^{\circ} \mathrm{C}$ PET is in glassy state and the modulated intensity shows the lowest amplitude. From about $80-100{ }^{\circ} \mathrm{C}$ (region II), PET is in a supercooled liquid state characterized by a slightly higher oscillation amplitude. In region III $\left(100-110{ }^{\circ} \mathrm{C}\right)$ the cold-crystallization occurred and the largest fluctuation is observed. Finally, above $110^{\circ} \mathrm{C}$ (region IV), the polymer was in a semi-crystalline state and the modulated intensity returned to oscillates with a constant amplitude.

A requirement to calculate properly the reversing component from the modulated signal is to achieve the linear response of the system during the MTFTIR experiment [6]. This condition can be checked from the observation of the Lissajous plots, in which the oscillating sample response, in this case the modulated intensity, is reported as function of the modulated temperature. In Fig. 4, the $\widetilde{\Delta I}_{1340}$ versus $\tilde{T}$ plots, obtained in the four time zones defined in Fig. 3, are displayed. They are related to the glassy state, the liquid state, the cold-crystallization process and to the semi-crystalline state of the PET sample.
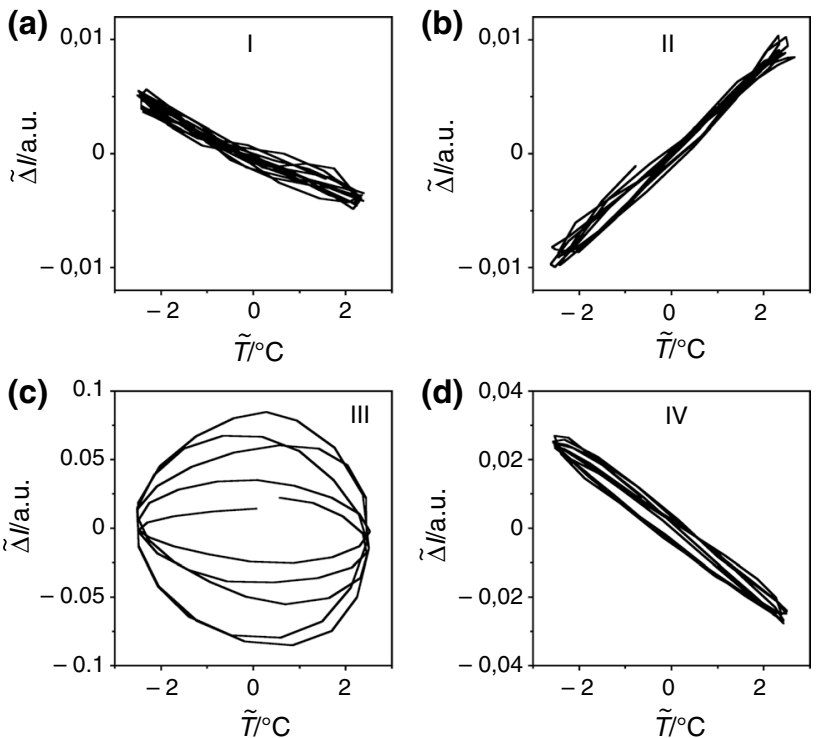

Fig. 4 Lissajous plots of modulated intensity of the $1340 \mathrm{~cm}^{-1}$ band $\left(\widetilde{\Delta I}_{1340}\right)$ as function of modulated temperature $(\tilde{T})$ of the PET sample in the glassy (a, I) and supercooled liquid state (b, II), during the cold-crystallization (c, III) and in the semi-crystalline state (d, IV)
In general, the linear response of the system is achieved in absence of ongoing kinetic processes and, therefore, the modulated intensity oscillates exclusively in-phase or antiphase with respect to the modulated temperature, being null the out-of-phase term of Eq. 8. Then, the Lissajous figure is expected to have a linear shape in which the cycles are superimposed, as observed in the glassy (Fig. 4a), liquid (Fig. 4b) and semi-crystalline polymer state (Fig. 4d). In these conditions, the linear slope of the cycles corresponds to the change of the reversing intensity with respect to temperature $\mathrm{d} I_{\vec{\nu}}^{\mathrm{R}} / \mathrm{d} T$, according to Eq. 9. Differently from modulated calorimetric measurement, in which the heat capacity is always positive, $\mathrm{d} I_{\bar{\nu}}^{\mathrm{R}} / \mathrm{d} T$ can assume either positive or negative values, depending on the behaviour of the specific absorption band and on the physical state of the material. As far as the $1340 \mathrm{~cm}^{-1}$ band, $\mathrm{d} I_{1340}^{\mathrm{R}} / \mathrm{d} T$ is negative in the glassy and semi-crystalline state, meanwhile it is positive in the supercooled liquid state. During the cold-crystallization, the linear response is obviously not preserved and, therefore, the Lissajous figures of different cycles do not overlap and become elliptic (Fig. 4c). When the system response is non-linear, a lack of accuracy of the reversing intensity determination occurs, as shown later.

In the next elaboration step, the temperature and intensity amplitudes $\left(A_{\mathrm{I}_{1340}}, A_{\mathrm{T}}\right)$ as well as the phase lag $(\varphi)$ were obtained from Eq. 13, 14 and 15. The results are reported in Fig. 5 as function of time and mean temperature. The complete dataset of elaborated data, comprising of temperature and intensity amplitudes, phase lags, as well as total, reversing and non-reversing intensities can be found in Online Resource 3.

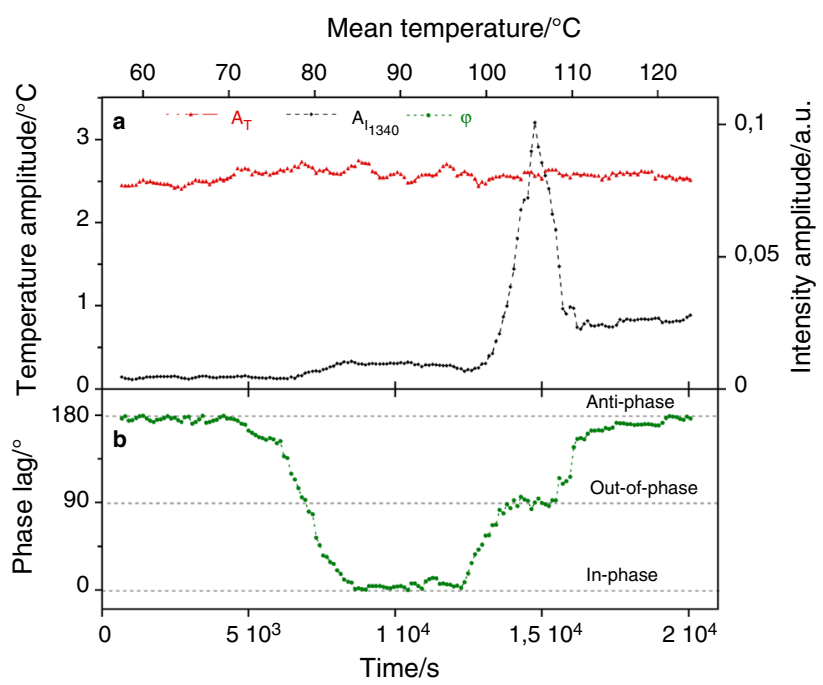

Fig. 5 Temperature amplitude (red triangles), intensity amplitude (black diamonds) of the band at $1340 \mathrm{~cm}^{-1}$ and phase lag (green circles) of quenched PET as function and time and mean temperature 
As aforementioned, the temperature amplitude remains constant around $2.5{ }^{\circ} \mathrm{C}$ in the whole temperature range (Fig. 5a). As far as the intensity amplitude, it is low and constant in the glassy state $\left(50-75^{\circ} \mathrm{C}\right)$ and, then, increases during the glass transition $\left(75-82^{\circ} \mathrm{C}\right)$ up to a constant value in the liquid state $\left(82-95^{\circ} \mathrm{C}\right)$. During the cold-crystallization, a peak was observed $\left(105^{\circ} \mathrm{C}\right)$. At the end, the semi-crystalline PET showed a constant and high intensity amplitude. It is interesting to note that the phase lag, calculated in angular degrees, ranges from $0^{\circ}$ to $180^{\circ}$. The intensity oscillates in anti-phase $\left(\varphi=180^{\circ}\right)$ respect to the temperature in the glassy as well as in the semi-crystalline state, and in-phase $\left(\varphi=0^{\circ}\right)$ in the liquid region. During the cold-crystallization, the out-of-phase behaviour $\left(90^{\circ}\right.$ phase lag $)$ is observed in correspondence of the intensity amplitude peak, as expected from the Lissajous figure (Fig. 4c).

According to the Eq. 9, the intensity and temperature amplitudes as well as the phase lag were used to calculate the real $\left(A_{\mathrm{I}_{1340}} \cos \varphi / A_{\mathrm{T}}\right)$ and imaginary $\left(A_{\mathrm{I}_{1340}} \sin \varphi / A_{\mathrm{T}}\right)$ components of the complex ratio between modulated intensity and temperature, reported in Fig. 6a as function of mean temperature. Moreover, the reversing intensity is displayed in Fig. 6b.

In correspondence to the glass transition of PET at $79^{\circ} \mathrm{C}$, the real component exhibits a rapid increase meanwhile a small peak can be observed in the imaginary component. As shown later in details, it is due to the recovery of the relaxation of the polymer glassy state from a non-equilibrium condition toward the liquid conformational equilibrium [34]. During the cold-crystallization, the real component decreases up to reach a constant negative value of the semi-crystalline state while the larger part of the oscillation

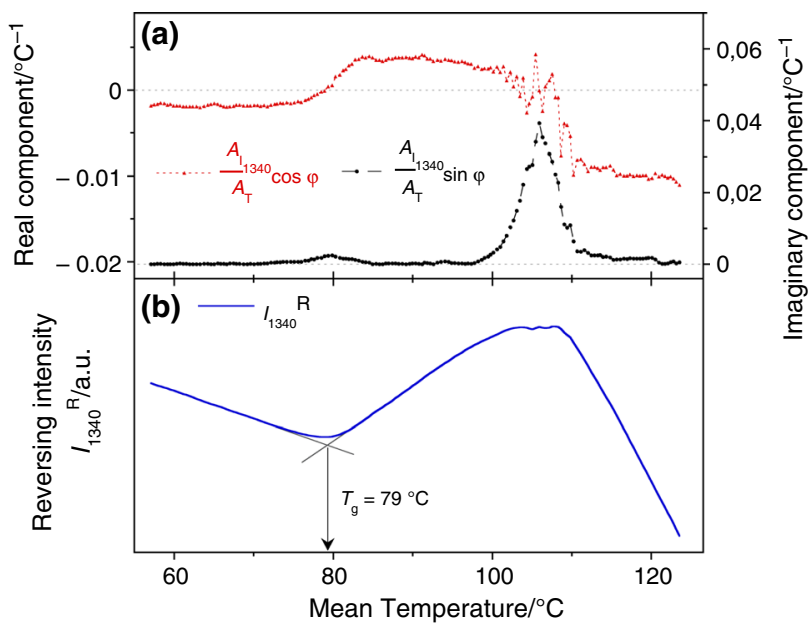

Fig. 6 a Real and imaginary parts of the ratio between complex intensity and complex temperature, calculated at the base frequency. b Reversing intensity of the $1340 \mathrm{~cm}^{-1}$ band as function of mean temperature amplitude is in the imaginary component (out-of-phase), evidenced by the peak centred at about $105{ }^{\circ} \mathrm{C}$. In this range, the non-linear effects of the cold-crystallization can be clearly observed in the real component by the appearance of spikes.

The reversing intensity $I_{1340}^{\mathrm{R}}$, obtained by the integration of the real component with respect to the mean temperature (Eq. 10), is reported in Fig. 6b. It shows a linear behaviour with two slope changes in correspondence to the glass transitions and crystallization. The dynamic glass transition temperature could be calculated precisely at the intersection of the extrapolated glass and liquid lines. At $0.2{ }^{\circ} \mathrm{C} \mathrm{min}^{-1}$ heating rate and at the base frequency of $0.0021 \mathrm{~Hz}(p=480 \mathrm{~s})$, it is located at $79^{\circ} \mathrm{C}$.

According to the Lambert-Beer law, the reversing intensity variation of the band at $1340 \mathrm{~cm}^{-1}$ with temperature $\left(\mathrm{d} I_{1340}^{\mathrm{R}} / \mathrm{d} T\right)$ depends on three factors, that are the reversing change of the optic path length $l$, of the molar extinction coefficient $\varepsilon$ (always reversible with temperature), and, more importantly, of the reversing trans ethylenic concentration $\left(\mathrm{d}[\mathrm{T}]^{\mathrm{R}} / \mathrm{d} T\right)$

$\frac{\mathrm{d} I_{1340}^{\mathrm{R}}}{\mathrm{d} T}=l \varepsilon \frac{\mathrm{d}[\mathrm{T}]^{\mathrm{R}}}{\mathrm{d} T}+l[\mathrm{~T}] \frac{\mathrm{d} \varepsilon}{\mathrm{d} T}+\varepsilon[\mathrm{T}] \frac{\mathrm{d} l}{\mathrm{~d} T}$

where $[\mathrm{T}]$ is the overall trans ethylenic conformer concentration that, analogously to the intensity, can be considered as a sum of a reversing $[\mathrm{T}]^{\mathrm{R}}$ and a non-reversing contribution $[\mathrm{T}]^{\mathrm{NR}}$. In the whole temperature range, it is assumed that the optic path length variation with temperature is negligible $(\mathrm{d} l / \mathrm{d} T=0)$.

In the glassy state $\left(60-80{ }^{\circ} \mathrm{C}\right), I_{1340}^{\mathrm{R}}$ decreases with increasing the mean temperature. In this region, the polymer chain mobility is very low and the conformational states are almost frozen in the time scale of the applied modulation frequency [16]. If $\mathrm{d}[\mathrm{T}]^{\mathrm{R}} / \mathrm{d} T=0$, then the reversing intensity variation is totally due to the change with temperature of the extinction coefficient, that generally decreases with increasing temperature $(\mathrm{d} \varepsilon / \mathrm{d} T<0)$. After the glass transition, the relaxation of polymer chains becomes much faster $[16,17]$, allowing conformational transitions to maintain the temperature dependent liquid equilibrium. In the case of PET, it was observed that in amorphous state the trans ethylenic conformation $(T)$ possesses a higher energy than that of the gauche $(G)[48,49]$. Hence, the temperature dependent conformational equilibrium, according to the Boltzmann distribution, favours the trans conformer by increasing the temperature $\left(\mathrm{d}[\mathrm{T}]^{\mathrm{R}} / \mathrm{d} T>0\right)$ [34]. The sum of the first two terms of the Eq. 16 leads to an increasing reversing intensity of the $1340 \mathrm{~cm}^{-1}$ band with temperature in the liquid region (Fig. 6b). Finally, after the crystallization $\left(T^{\mathrm{M}}>110^{\circ} \mathrm{C}\right)$ the trans concentration increased drastically because of the formation of all-trans sequences typical of the crystal structure 
of PET. In this case, the positive first term of the Eq. 16, representing the conformational liquid equilibrium of the residual amorphous fraction, decreases, meanwhile the negative second term increases in absolute value because of the huge increase of the overall trans ethylenic concentration [T]. Indeed, the resulting reversing intensity in the semi-crystalline region $\left(>110^{\circ} \mathrm{C}\right)$ shows a steeper negative slope than that of the glassy region, in which the overall trans concentration is low.

The subtraction of the reversing component to the total intensity gives the non-reversing intensity, which contains the information of the non-equilibrium processes or/and of those transformation that are too slow to be reversed in a period of modulation. In Fig. 7, the total intensity as well as the reversing and non-reversing components are compared as function of mean temperature. The reversing and nonreversing intensities are arbitrarily shifted for the sake of clarity.

The non-reversing intensity variation with temperature can be expressed, analogously to the reversing intensity, by Eq. 17, representing the non-equilibrium processes, such as the conformational relaxations in the amorphous state and the formation of the crystalline phase.

$\frac{\mathrm{d} I_{1340}^{\mathrm{NR}}}{\mathrm{d} T}=l \varepsilon \frac{\mathrm{d}[\mathrm{T}]^{\mathrm{NR}}}{\mathrm{d} T}$

Hence, the non-reversing intensity variation with temperature of the $1340 \mathrm{~cm}^{-1}$ band is due exclusively to the nonequilibrium concentration change with temperature of the trans ethylenic conformer $\left(\mathrm{d}[\mathrm{T}]^{\mathrm{NR}} / \mathrm{d} T\right)$. Starting from low temperature, $I_{1340}^{\mathrm{NR}}$ remains constant between 50 and $75^{\circ} \mathrm{C}$, temperature range in which the glassy state did not undergo any appreciable transformation. At higher temperature,

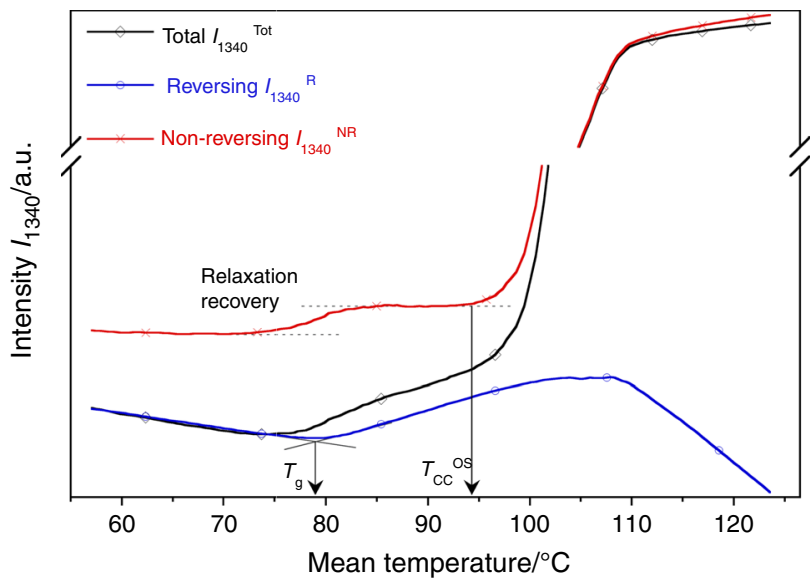

Fig. 7 Total ( $I_{1340}^{\text {Tot }}$, black line, open diamonds), reversing $\left(I_{1340}^{\mathrm{R}}\right.$, blue line, open circles) and non-reversing intensity $\left(I_{1340}^{\mathrm{NR}}\right.$, red line, crosses) of the $1340 \mathrm{~cm}^{-1}$ band as a function of mean temperature the increase of the non-reversing intensity between 75 and $85^{\circ} \mathrm{C}$, superimposed to the glass transition, is associated to the relaxation recovery [34,57], consisting of the gauche to trans transition of a small fraction of ethylenic conformers. It occurs because the polymer slowly relaxes in the glassy state towards a more stable structure by increasing gauche conformer concentration. This relaxation takes place mainly during the slow cooling at $2{ }^{\circ} \mathrm{C} \mathrm{min}{ }^{-1}$ from 82 to $50{ }^{\circ} \mathrm{C}$, before the MTFTIR experiment. As soon as the chains acquired enough mobility at $T_{\mathrm{g}}$, the macromolecular chains recovered the liquid equilibrium. In the supercooled liquid region $\left(85-95^{\circ} \mathrm{C}\right)$, non-equilibrium relaxations involving ethylenic conformers $(\mathrm{T}, \mathrm{G})$ are absent, being constant the non-reversing intensity. Then, the primary cold-crystallization process starts at about $94{ }^{\circ} \mathrm{C}$ with a $I_{1340}^{\mathrm{NR}}$ steep increase, up to $110^{\circ} \mathrm{C}$. At higher temperature, the further increase of the signal is due to the slow secondary crystallization.

The advantage of temperature or time resolved infrared spectroscopy over other thermal analysis was exploited by following the intensity changes with temperature of other bands, related to different conformers or conformer sequences. In particular, the intensity variation of the absorption at $971 \mathrm{~cm}^{-1}$ with temperature has been chosen (Fig. 2b). This vibration is assigned to the $\mathrm{O}-\mathrm{CH}_{2}$ stretching mode of the ethylene glycol conformer belonging to a planar structure [42, 51]. Moreover, it has been found that its intensity is directly correlated with the PET crystallinity [58] and, then, the absorption can be likely associated to the all-trans sequence $\left(\mathrm{TtT}_{\mathrm{B}}\right)$ of the PET repeating unit. By performing the elaboration procedure previously reported, the total, reversing and non-revering intensities for the $971 \mathrm{~cm}^{-1}$ band were calculated and reported as function of mean temperature in Fig. 8.

As shown in the spectra of Fig. 2, the $971 \mathrm{~cm}^{-1}$ band, although weak, was already present in the amorphous PET, as observed in other studies [41,49-51]. The general features of the band intensity variation are very similar to that of the band at $1340 \mathrm{~cm}^{-1}$, showing the glass transition at $79^{\circ} \mathrm{C}$, the relaxation recovery between 75 and $85^{\circ} \mathrm{C}$ and the cold-crystallization onset $\left(T_{\mathrm{cc}}^{\mathrm{os}}\right)$ at about $94{ }^{\circ} \mathrm{C}$. On the other hand, the non-reversing intensity component $\left(I_{971}^{\mathrm{NR}}\right)$ shows some differences in the temperature range where the polymer is in the supercooled liquid state, that is between $T_{\mathrm{g}}$ and $T_{\mathrm{cc}}^{\mathrm{os}}\left(80-94{ }^{\circ} \mathrm{C}\right)$. In fact, soon after the relaxation recovery, $I_{971}^{\mathrm{NR}}$ continues to increase stepwise with temperature whereas $I_{1340}^{\mathrm{NR}}$ levelled off (Fig. 7). To better appreciate the difference between the two behaviours, the non-reversing intensity of the analysed bands are displayed together as function of mean temperature in Fig. 9. For sake of comparison, their respective intensities, very different in the original spectra, were normalized between 0 and 1 at $60^{\circ} \mathrm{C}$ and $115^{\circ} \mathrm{C}$, respectively. 


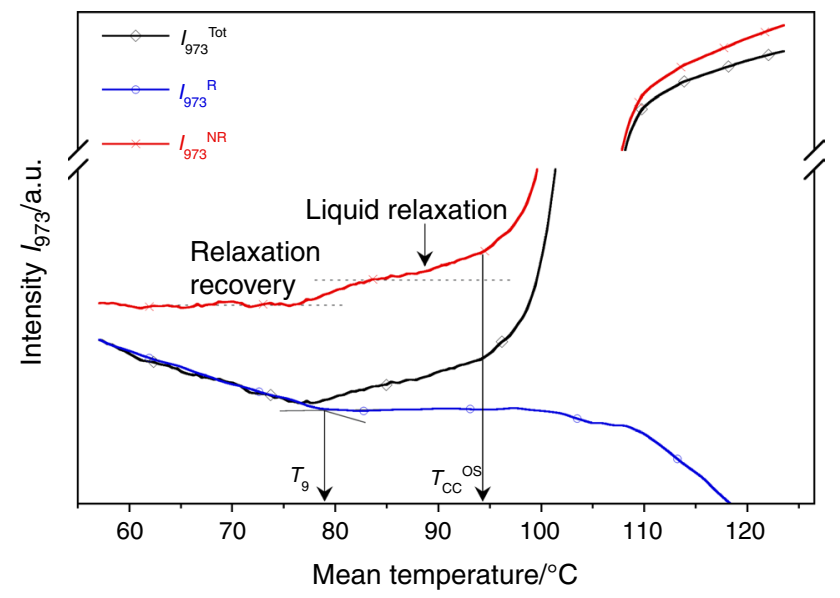

Fig. 8 Total ( $I_{971}^{\text {Tot }}$, black line, open diamonds), reversing ( $I_{971}^{\mathrm{R}}$, blue line, open circles) and non-reversing intensity ( $I_{971}^{\mathrm{NR}}$, red line, crosses) of the $971 \mathrm{~cm}^{-1}$ band of quenched PET as a function of mean temperature
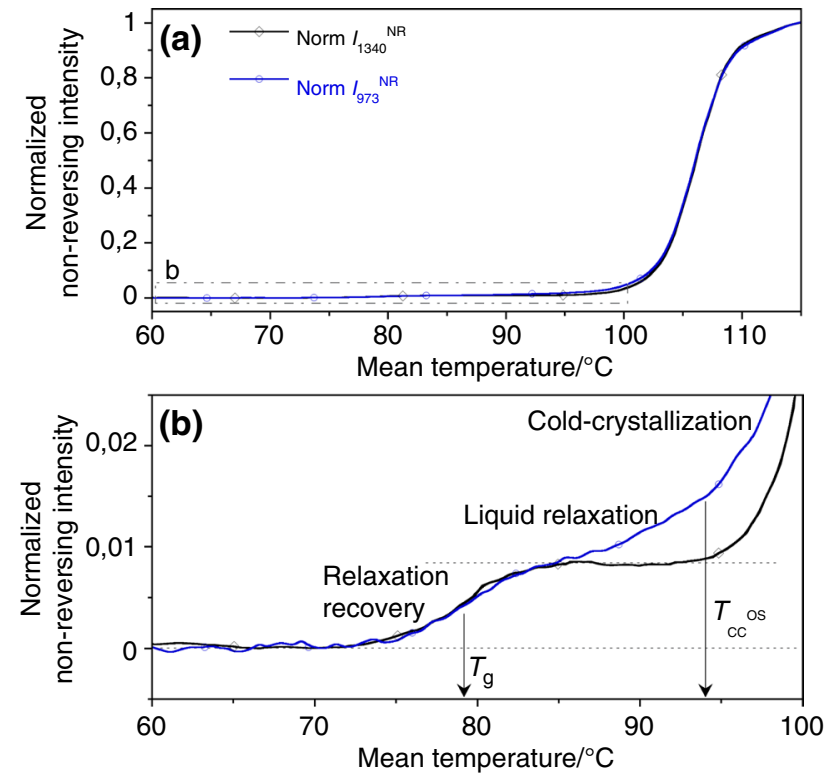

Fig. 9 a Comparison between normalized non-reversing intensities of $1340 \mathrm{~cm}^{-1}$ and $971 \mathrm{~cm}^{-1}$ bands as function of mean temperature. b Enlargement of the glassy and liquid region. At $T_{\mathrm{g}}=79^{\circ} \mathrm{C}$ and $T_{\mathrm{cc}}^{\mathrm{os}}=94^{\circ} \mathrm{C}$, the glass transition and cold-crystallization onset were marked by vertical arrows

Similarly to $I_{1340}^{\mathrm{NR}}$, it is possible to observe clearly the increase of $I_{971}^{\mathrm{NR}}$ between 75 and $85^{\circ} \mathrm{C}$, due to the relaxation recovery (Fig. 9b). The variation of the normalized intensity the two bands in correspondence to the recovery process is equal because the gauche to trans transition of the ethylenic unit $(\mathrm{G} \rightarrow \mathrm{T})$, observed at $1340 \mathrm{~cm}^{-1}$, leads to the formation of $\mathrm{TtT}_{\mathrm{B}}$ sequences, absorbing at $971 \mathrm{~cm}^{-1}$.
The further interpretation of the phenomena was carried out by referring to the NMR, IR and Raman studies on conformer distribution in the PET amorphous state reported by Stokr et al. [49]. It has been observed that one of the most probable conformation assumed by the repeating unit of PET in the amorphous phase is the $\mathrm{GtT}_{\mathrm{B}}$. So, the concordance of the normalized $I_{1340}^{\mathrm{NR}}$ and $I_{971}^{\mathrm{NR}}$ values in the relaxation recovery region could be accounted for the transition of a small fraction of $\mathrm{GtT}_{\mathrm{B}}$ sequences into $\mathrm{TtT}_{\mathrm{B}}$. The same approach could be used to explain the non-reversing intensity increase of the $971 \mathrm{~cm}^{-1}$ band in the supercooled liquid region $\left(85-94^{\circ} \mathrm{C}\right)$. It is caused by the non-reversing formation of a small fraction of $\mathrm{TtT}_{\mathrm{B}}$ sequences in the liquid state, probably driven by the more energetically favourable crystalline phase. This happens while the $I_{1340}^{\mathrm{NR}}$ remains constant up to the coldcrystallization onset $\left(T_{\mathrm{cc}}^{\mathrm{ss}}=94{ }^{\circ} \mathrm{C}\right)$, evidencing that the ethylenic $\mathrm{CH}_{2}-\mathrm{CH}_{2}$ bond is not involved in any conformational transitions of the polymer in the supercooled liquid state. It means that, at least in the used experimental conditions, the two possible ethylenic conformers reached the metastable equilibrium after the recovery. Hence, the all-trans sequence formation could be caused by the gauche-trans glycolic transition $(\mathrm{g} \rightarrow \mathrm{t})$ or by the cis-trans terephthalic transition $\left(\mathrm{C}_{\mathrm{B}} \rightarrow \mathrm{T}_{\mathrm{B}}\right)$. On the basis of spectral evidences, all those repeating units that possessed the trans form of the ethylenic unit $(\mathrm{T})$ could be involved in the liquid relaxation. Among them, the $\mathrm{TtC}_{\mathrm{B}}$ is the most abundant sequence (the others having the glycol unit in gauche, such as $\mathrm{TgC}_{\mathrm{B}}$ and $\mathrm{TgT}_{\mathrm{B}}$, are reported to be less favourable [49-51]) and its non-reversing transition could be considered the main route of the liquid relaxation. Unfortunately, more direct mechanism interpretation cannot be done because the intensity variation of other bands sensitive to glycolic ( $g$ or $t$ ) or terephthalic $\left(\mathrm{C}_{\mathrm{B}}\right.$ or $\left.\mathrm{T}_{\mathrm{B}}\right)$ conformations is too weak to be monitored in the employed experimental conditions. However, besides this, it can be inferred that the PET supercooled liquid state is characterized by a micro-heterogeneity in that the isolated ethylenic conformers are in-equilibrium while the conformational state of the entire repeating unit is in non-equilibrium. On the other hand, structural organization phenomena of PET prior to the cold-crystallization have been already observed $[45,47]$. Nonetheless, MTFTIR has proved to be a suitable technique to observe non-reversing transformation occurring in the PET supercooled liquid, able to give information complementary to that acquired with other thermal analysis. Further investigation on this issue are now in progress.

Finally, above $94{ }^{\circ} \mathrm{C}$, the cold-crystallization of PET took place, as evidenced by the rapid and simultaneous increase of the non-reversing intensities of the two bands (Fig. 9a). 


\section{Conclusions}

The thermal behaviour of materials can be studied by different techniques. The time or temperature resolved FTIR offers the opportunity to follow material transition at microstructural level. As far as polymer, the different local conformations or conformation sequences the macromolecules can assume give rise to specific bands whose intensity changes according to structural evolution. In this paper, the implementation of the temperature resolved FTIR analysis, obtained by adding to the conventional constant heating or cooling rate a temperature modulation, was described. In particular, the theoretical background, the experimental set up, the step-by-step data elaboration of the data acquired by MTFTIR analysis was reported. By using the new investigation method, it has been possible to separate the equilibrium and non-equilibrium conformational rearrangements of PET in the temperature range of the glass transition and cold-crystallization. In order to show the advantages of the proposed technique, the data elaboration was performed on two different absorption bands of PET, the $1340 \mathrm{~cm}^{-1}$ and $971 \mathrm{~cm}^{-1}$, related to the trans ethylenic conformation (T) and the all-trans sequence $\left(\mathrm{TtT}_{\mathrm{B}}\right)$ respectively. At the glass transition $\left(T_{\mathrm{g}}=79^{\circ} \mathrm{C}\right)$ a slope change in the reversing intensity of both bands was observed as a function of the mean temperature. Then, at higher temperatures, the steplike increase of the non-reversing intensity was attributed to the non-equilibrium relaxation recovery given by the conformational transition of ethylenic conformers from the gauche to the trans form $(\mathrm{G} \rightarrow \mathrm{T})$. In the supercooled liquid region $\left(85-94{ }^{\circ} \mathrm{C}\right)$, a non-reversing transformation was observed exclusively for the $971 \mathrm{~cm}^{-1}$ band, suggesting an increase of repeating units in all-trans structure, presumably triggered by the cis to trans conformational transition of terephthalic units $\left(C_{B} \rightarrow T_{B}\right)$. The non-reversible transformations occurring in the temperature region where the polymer is in the supercooled state, clearly identified by the MTFTIR analysis, could be the key processes which predispose the polymer for the following cold-crystallization at $94{ }^{\circ} \mathrm{C}$. It is evidenced by the largest increase of non-reversing intensity of both bands, related to the conformational ordering of PET chains in all-trans structures. On the basis of the findings reported in the present research, the authors believe that a detailed investigation of the conformer evolution at $T>T_{\mathrm{g}}$ is an intriguing issue which can give further indication on polymer rearrangement before the structural ordering.

Acknowledgements The authors acknowledge the financial support of Sapienza University of Rome.

Authors' contribution All authors contributed to the study conception and design, with the supervision of Prof. Andrea Martinelli. All authors contributed equally to the methodology, software, material preparation, data collection and investigation. The first draft of the manuscript was written by Valerio Di Lisio and all authors commented on previous versions of the manuscript. All authors read and approved the final manuscript.

Funding The project was funded by "Progetti per Avvio alla Ricerca di Ateneo 2019" (Grant No. AR21916B8889E074) and "Progetti di Ricerca di Ateneo 2018" (Grant No. RM11816435F88EF6). Open access funding provided by Università degli Studi di Roma La Sapienza within the CRUI-CARE Agreement.

Availability of data and material This paper has datasets included in the Electronic Supplementary Material.

\section{Compliance with ethical standards}

Conflict of interest The authors declare that they have no conflict of interest.

Ethical approval This article does not contain any studies with human participants or animals performed by any of the authors.

Open Access This article is licensed under a Creative Commons Attribution 4.0 International License, which permits use, sharing, adaptation, distribution and reproduction in any medium or format, as long as you give appropriate credit to the original author(s) and the source, provide a link to the Creative Commons licence, and indicate if changes were made. The images or other third party material in this article are included in the article's Creative Commons licence, unless indicated otherwise in a credit line to the material. If material is not included in the article's Creative Commons licence and your intended use is not permitted by statutory regulation or exceeds the permitted use, you will need to obtain permission directly from the copyright holder. To view a copy of this licence, visit http://creativecommons.org/licenses/by/4.0/.

\section{References}

1. Charsley E, Price D, Hunter N, Gabbott P, Kett V, Gaisford S, Parkes G. Principles of thermal analysis and calorimetry. Royal society of chemistry; 2019.

2. Haines PJ. Symultaneous techniques and product analysis. In: Haines PJ, editor. Thermal methods of analysis: principles, applications and problems. Springer, Berlin; 2012. pp. 161-205.

3. Vyazovkin S, Nobuyoshi K, Schick C. Handbook of thermal analysis and Calorimetry: Recent Advances, techniques and applications. Vol. 6. Elsevier; 2018.

4. Gill PS, Sauerbrunn SR, Reading M. Modulated differential scanning calorimetry. J Therm Anal. 1993. https://doi.org/10.1007/ BF02546852.

5. Schawe JEK, Höhne GWH. The analysis of temperature modulated DSC measurements by means of the linear response theory. Thermochim Acta. 1996. https://doi.org/10.1016/00406031(96)88984-2.

6. Schawe JEK. Principles for the interpretation of modulated temperature DSC measurements. Part 1. Glass transition. Thermochim Acta. 1995. https://doi.org/10.1016/0040-6031(95)02315-S.

7. Lacey AA, Price DM, Reading M. Theory and practice of modulated temperature differential scanning calorimetry. In: Reading M, Hourston DJ, editors. Modulated Temperature Differential Scanning Calorimetry. Hot Topics in Thermal Analysis and Calorimetry, vol 6. Springer, Dordrecht; 2006. pp 1-81. 
8. Okazaki I, Wunderlich B. Reversible melting in polymer crystals detected by temperature-modulated differential scanning calorimetry. Macromolecules. 1997. https://doi.org/10.1021/ma961539d.

9. Wunderlich B, Okazaki I. Modulated differential scanning calorimetry in the glass transition region. J Therm Anal. 1997. https ://doi.org/10.1007/BF01987421.

10. Toda A, Arita T, Tomita C, Hikosaka M. Temperature-modulated DSC applied to the transformation kinetics of polymer crystallization. Polym J. 1999. https://doi.org/10.1295/polymj.31.790.

11. Androsch R, Wunderlich B. Specific reversible melting of polyethylene. J Polym Sci Pol Phys. 2003. https://doi.org/10.1002/ polb.10574.

12. Pak J, Wunderlich B. Reversible melting of polyethylene extendedchain crystals detected by temperature-modulated calorimetry. J Polym Sci Pol Phys. 2002. https://doi.org/10.1002/polb.10283.

13. Androsch R, Wunderlich B. Reversible crystallization and melting at the lateral surface of isotactic polypropylene crystals. Macromolecules. 2001. https://doi.org/10.1021/ma010260g.

14. Genovese A, Shanks RA. Crystallization and melting of isotactic polypropylene in response to temperature modulation. $\mathrm{J}$ Therm Anal Calorim. 2004. https://doi.org/10.1023/B:JTAN.0000017345 $.31134 .8 \mathrm{~d}$

15. Di Lorenzo ML. Calorimetric analysis of the multiple melting behavior of poly (L-lactic acid). J Appl Polym Sci. 2006. https:// doi.org/10.1002/app.23136.

16. Hutchinson J. Determination of the glass transition temperature: methods correlation and structural heterogeneity. J Therm Anal Calorim. 2009. https://doi.org/10.1007/s10973-009-0268-0.

17. Monnier X, Cangialosi D. Effect of molecular weight on vitrification kinetics and molecular mobility of a polymer glass confined at the microscale. Thermochim Acta. 2019. https://doi. org/10.1016/j.tca.2019.01.011.

18. Schick C, Merzlyakov M, Minakov A, Wurm A. Crystallization of polymers studied by temperature modulated calorimetric measurements at different frequencies. J Therm Anal Calorim. 2000. https ://doi.org/10.1023/A:1010108617592.

19. Righetti MC, Laus M, Di Lorenzo ML. Temperature dependence of the rigid amorphous fraction in poly (ethylene terephthalate). Eur Polym J. 2014. https://doi.org/10.1016/j.eurpo lymj.2014.06.005.

20. Schawe JEK, Pogatscher S. Material characterization by fast scanning calorimetry: practice and applications. In: Schick C, Mathot V, editors. Fast scanning calorimetry. Springer: Cham; 2016. p. 3-80.

21. Toda A, Androsch R, Schick C. Insights into polymer crystallization and melting from fast scanning chip calorimetry. Polymer. 2016. https://doi.org/10.1016/j.polymer.2016.03.038.

22. Shoifet E, Schulz G, Schick C. Temperature modulated differential scanning calorimetry-extension to high and low frequencies. Termochim Acta. 2015. https://doi.org/10.1016/j.tca.2014.10.010.

23. Perez-de-Eulate NG, Di Lisio V, Cangialosi D. Glass transition and molecular dynamics in polystyrene nanospheres by fast scanning calorimetry. ACS Macro Lett. 2017. https://doi.org/10.1021/ acsmacrolett.7b00484.

24. Price DM. Modulated-temperature thermomechanical analysis. J Therm Anal Calorim. 1998. https://doi.org/10.1007/BF02719024.

25. Efremov MY, Kiyanova AV, Nealey PF. Temperature-modulated ellipsometry: a new probe for glass transition in thin supported polymer films. Macromolecules. 2008. https://doi.org/10.1021/ ma8011416.

26. Wurm A, Merzlyakov M, Schick C. Reversible melting during crystallization of polymers studied by temperature modulated techniques (TMDSC, TMDMA). J Thermal Anal Calorim. 2000. https://doi.org/10.1023/A:1010195321797.
27. Blaine RL, Hahn BK. Obtaining kinetic parameters by modulated thermogravimetry. J Therm Anal Calorim. 1998. https://doi. org/10.1023/A:1010171315715.

28. Goderis B, Reynaers H, Scherrenberg R, Mathot VB, Koch MH. Temperature reversible transitions in linear polyethylene studied by TMDSC and time-resolved, temperature-modulated WAXD/ SAXS. Macromolecules. 2001. https://doi.org/10.1021/ma001 $759 \mathrm{y}$.

29. Oka Y, Yao H, Saruyama Y. Temperature-modulated X-ray diffractometry applied to a study on calorimetric and structural change of semicrystalline poly (ethylene oxide). J Therm Anal Calorim. 2016. https://doi.org/10.1007/s10973-016-5235-y.

30. Honda H. In situ temperature-modulated microscopy observation of two-phase co-existing states of coumarin and coumarin derivative in melting. J Therm Anal Calorim. 2019. https://doi. org/10.1007/s10973-018-7952-x.

31. Gouveia JR, Vidotti SE, Augusto AC, Tavares LB, dos Santos DJ. Temperature modulated optical refractometry. J Therm Anal Calorim. 2019. https://doi.org/10.1007/s10973-019-08797-2.

32. Caminiti R, D'Ilario L, Martinelli A, Piozzi A. Poly (p-phenylene sulfide) isothermal cold crystallization investigated by usual and unusual methods. Macromol Chem Phys. 2001. https://doi. org/10.1002/1521-3935(20011001)202:14\%3c2902:AID-MACP2 $902 \% 3 \mathrm{e} 3.0 . \mathrm{CO} ; 2-\mathrm{V}$

33. Lv R, Na B, Tian N, Zou S, Li Z, Jiang S. Mesophase formation and its thermal transition in the stretched glassy polylactide revealed by infrared spectroscopy. Polymer. 2011. https://doi. org/10.1016/j.polymer.2011.08.023.

34. Wang Y, Shen D, Qian R. Subglass-transition-temperature annealing of poly (ethylene terephthalate) studied by FTIR. J Polym Sci Pol Phys. 1998. https://doi.org/10.1002/(SICI)1099-0488(19980 415)36:5\%3c783:AID-POLB5\%3e3.0.CO;2-M.

35. Zhang J, Duan Y, Sato H, Tsuji H, Noda I, Yan S, Ozaki Y. Crystal modifications and thermal behavior of poly (L-lactic acid) revealed by infrared spectroscopy. Macromolecules. 2005. https ://doi.org/10.1021/ma051232r.

36. Baurecht D, Fringeli UP. Quantitative modulated excitation Fourier transform infrared spectroscopy. Rev Sci Instrum. 2001. https ://doi.org/10.1063/1.1400152.

37. Müller M, Buchet R, Fringeli UP. 2D-FTIR ATR spectroscopy of thermo-induced periodic secondary structural changes of poly-(L)lysine: a cross-correlation analysis of phase-resolved temperature modulation spectra. J Phys Chem. 1996. https://doi.org/10.1021/ jp9602843.

38. Chandran S, Baschnagel J, Cangialosi D, Fukao K, Glynos E, Janssen LM, Müller M, Muthukumar M, Steiner U, Xu J, Napolitano S, Reiter G. Processing pathways decide polymer properties at the molecular level. Macromolecules. 2019. https://doi. org/10.1021/acs.macromol.9b01195.

39. Di Lisio V, Sturabotti E, Francolini I, Piozzi A, Martinelli A. Effects of annealing above $\mathrm{Tg}$ on the physical aging of quenched PLLA studied by modulated temperature FTIR. J Polym Sci Pol Phys. 2019. https://doi.org/10.1002/polb.24769.

40. Di Lisio V, Sturabotti E, Francolini I, Piozzi A, Martinelli A. Isotactic polypropylene reversible crystallization investigated by modulated temperature and quasi-isothermal FTIR. J Polym Sci Pol Phys. 2019. https://doi.org/10.1002/polb.24847.

41. Chen Z, Hay JN, Jenkins MJ. The thermal analysis of poly (ethylene terephthalate) by FTIR spectroscopy. Thermochim Acta. 2013. https://doi.org/10.1016/j.tca.2012.11.002.

42. Yoshii T, Yoshida H, Kawai T. Effect of structural relaxation of glassy PET on crystallization process observed by the simultaneous DSC-XRD and DSC-FTIR. Thermochim Acta. 2005. https ://doi.org/10.1016/j.tca.2005.01.070.

43. Sathyanarayana PM, Shariff G, Thimmegowda MC, Ashalatha MB, Ramani R, Ranganathaiah C. Structural relaxation in poly 
(ethylene terephthalate) studied by positron annihilation lifetime spectroscopy. Polym Int. 2002. https://doi.org/10.1002/pi.924.

44. Dobbertin J, Hensel A, Schick C. Dielectric spectroscopy and calorimetry in the glass transition region of semi-crystalline poly (ethylene terephthalate). J Therm Anal Calorim. 1996. https://doi. org/10.1007/BF01979446.

45. Huang JM, Chu PP, Chang FC. Conformational changes and molecular motion of poly (ethylene terephthalate) annealed above glass transition temperature. Polymer. 2000. https://doi. org/10.1016/S0032-3861(99)00329-8.

46. Karagiannidis PG, Stergiou AC, Karayannidis GP. Study of crystallinity and thermomechanical analysis of annealed poly (ethylene terephthalate) films. Eur Polym J. 2008. https://doi. org/10.1016/j.eurpolymj.2008.02.024.

47. Imai M, Kaji K, Kanaya T, Sakai Y. Ordering process in the induction period of crystallization of poly (ethylene terephthalate). Phys Rev B. 1995. https://doi.org/10.1103/PhysRevB.52.12696.

48. Eslami H, Müller-Plathe F. Structure and mobility of poly (ethylene terephthalate): a molecular dynamics simulation study. Macromolecules. 2009. https://doi.org/10.1021/ma901780e.

49. Štokr J, Schneider B, Doskočilová D, Lövy J, Sedláček P. Conformational structure of poly (ethylene terephthalate). Infra-red, Raman and nmr spectra. Polymer. 1982; https://doi. org/10.1016/0032-3861(82)90057-X.

50. Cole KC, Ajji A, Pellerin E. New insights into the development of ordered structure in poly (ethylene terephthalate). 1. Results from external reflection infrared spectroscopy. Macromolecules. 2002; https://doi.org/10.1021/ma011492i.

51. Cole KC, Ajji A, Pellerin E. New insights into the development of ordered structure in poly (ethylene terephthalate), II Results from transmission infrared spectroscopy of thin films.
Macromol Symp. 2002. https://doi.org/10.1002/1521-3900(20020 8) 184:1\%3c1:AID-MASY1\%3e3.0.CO;2-O.

52. Melveger AJ. Laser-raman study of crystallinity changes in poly (ethylene terephthalate). J Polym Sci A2. 1972; https://doi. org/10.1002/pol.1972.160100211.

53. Purvis J, Bower DI. Molecular orientation in poly (ethylene terephthalate) by means of laser-raman spectroscopy. J Polym Sci Polym Phys Edn. 1976. https://doi.org/10.1002/pol.1976.18014 0809.

54. Daubeny RDP, Bunn CW, Brown CJ. The crystal structure of polyethylene terephthalate. Proc. R. Soc. Lond. Ser. A. Math. Phys. Sci. 1954; https://doi.org/10.1098/rspa.1954.0273.

55. Kifliea K, Piccarolo S, Brucato V, Baltá-Calleja FJ. Role of thermal history on quiescent cold crystallization of PET. Polymer. 2002. https://doi.org/10.1016/S0032-3861(02)00260-4.

56. Gaur U, Lau SF, Wunderlich BB, Wunderlich B. Heat capacity and other thermodynamic properties of linear macromolecules. VIII. Polyesters and polyamides. J Phys Chem Ref Data. 1983. https://doi.org/10.1063/1.555678.

57. Hodge IM. Enthalpy relaxation and recovery in amorphous materials. J Non-Cryst Solids. 1994. https://doi.org/10.1016/00223093(94)90321-2.

58. Yazdanian M, Ward IM, Brody H. An infra-red study of the structure of oriented poly (ethylene terephthalate) fibres. Polymer. 1985. https://doi.org/10.1016/0032-3861(85)90003-5.

Publisher's Note Springer Nature remains neutral with regard to jurisdictional claims in published maps and institutional affiliations. 